\title{
Ability of artificial intelligence to diagnose coronary artery stenosis using hybrid images of coronary computed tomography angiography and myocardial perfusion SPECT
}

Hiroto Yoneyama ${ }^{1 *} \mathbb{D}$, Kenichi Nakajima², Junichi Taki ${ }^{2}$, Hiroshi Wakabayashi ${ }^{2}$, Shinro Matsuo ${ }^{2}$, Takahiro Konishi Koichi Okuda ${ }^{3}$, Takayuki Shibutani ${ }^{4}$, Masahisa Onoguchi ${ }^{4}$ and Seigo Kinuya ${ }^{2}$

\footnotetext{
* Correspondence: kizu@cf6.so-net. ne.jp

'Department of Radiological Technology, Kanazawa University Hospital, 13-1 Takara-machi, Kanazawa 920-8641, Japan Full list of author information is available at the end of the article
}

\begin{abstract}
Background: Detecting culprit coronary arteries in patients with ischemia using only myocardial perfusion single-photon emission computed tomography (SPECT) can be challenging. This study aimed to improve the detection of culprit regions using an artificial neural network (ANN) to analyze hybrid images of coronary computed tomography angiography (CCTA) and myocardial perfusion SPECT.

Methods: This study enrolled 59 patients with stable coronary artery disease (CAD) who had been assessed by coronary angiography within 60 days of myocardial perfusion SPECT. Two nuclear medicine physicians interpreted the myocardial perfusion SPECT and hybrid images with four grades of confidence, then drew regions on polar maps to identify culprit coronary arteries. The gold standard was determined by the consensus of two other nuclear cardiology specialist based on coronary angiography findings and clinical information. The ability to detect culprit coronary arteries was compared among experienced nuclear cardiologists and the ANN. Receiver operating characteristics (ROC) curves were analyzed and areas under the ROC curves (AUC) were determined.
\end{abstract}

Results: Using hybrid images, observer A detected CAD in the right (RCA), left anterior descending (LAD), and left circumflex (LCX) coronary arteries with $83.6 \%$, $89.3 \%$, and $94.4 \%$ accuracy, respectively and observer B did so with $72.9 \%, 84.2 \%$, and $89.3 \%$, respectively. The ANN was $79.1 \%, 89.8 \%$, and $89.3 \%$ accurate for each coronary artery. Diagnostic accuracy was comparable between the ANN and experienced nuclear medicine physicians. The AUC was significantly improved using hybrid images in the $\mathrm{RCA}$ region (observer $\mathrm{A}$ : from 0.715 to $0.835, p=0.0031$; observer $\mathrm{B}$ : from 0.771 to $0.843, p=0.042$. To detect culprit coronary arteries in perfusion defects of the inferior wall without using hybrid images was problematic because the perfused areas of the LCX and RCA varied among individuals.

Conclusions: Hybrid images of CCTA and myocardial perfusion SPECT are useful for detecting culprit coronary arteries. Diagnoses using artificial intelligence are comparable to that by nuclear medicine physicians.

Keywords: Cardiac evaluation, Nuclear cardiology, Ischemia, Computer-aided diagnosis, Neural network 


\section{Background}

Myocardial perfusion imaging (MPI) is widely used to noninvasively assess reversible myocardial ischemia (Henzlova et al. 2016; Matsumoto and Hirayama 2017; Kiso et al. 2017; Matsuda and Takeishi 2016). Hachmovitch et al. reported that $10 \%$ ischemia of the left ventricle can be used as a guide to effective coronary revascularization, and a Japanese multicenter study also demonstrated that $\geq 5 \%$ of ischemic reduction led the improvement of patient outcome (Hachmovitch et al. 2003; Shaw et al. 2008; Nanasato et al. 2018). While importance of the assessment of ischemia is included in nuclear cardiology guidelines at present, all the institutions do not necessarily have nuclear cardiology specialists. Artificial intelligence (AI) has also been applied to nuclear cardiology and might aid in the interpretation of single-photon emission computed tomography (SPECT) images (Arsanjani et al. 2013a; Motowani et al. 2016; Arsanjani et al. 2013b; Arsanjani et al. 2015; Guner et al. 2010; Nakajima et al. 2018; Nakajima et al. 2017; Nakajima et al. 2015; Johansson et al. 2014). Since the AI can identify possible abnormal regions with a degree of probability, it should be important to clarify how we can use AI for the clinical practice. On the other hand, although coronary angiography is regarded as the gold standard for a diagnosis of coronary artery disease (CAD), it is invasive and not the first choice for assessing patients. Thus, coronary computed tomography angiography (CCTA) is rapidly gaining clinical acceptance (Kiriyama et al. 2018), and it complements myocardial perfusion SPECT (MP-SPECT) in the assessment of CAD (Levine et al. 2011; Sato et al. 2015). Kirisli et al. reported that hybrid images of MP-SPECT and CCTA offered the additional diagnostic benefit of allowing myocardial perfusion defect correlations with corresponding coronary arteries (Kirisli et al. 2014). Furthermore, Slomka et al. reported that CTA-guided MPI analysis correctly identified stenotic lesions that had been confirmed by invasive angiography, particularly in the right coronary (RCA) and left circumflex (LCX) arteries, whereas CTA alone and MPI unaided by CTA did not (Slomka et al. 2009). Several detailed studies have investigated the clinical usefulness of hybrid images. However, none have focused on interpreting hybrid images using cardioREPO/EXINI Heart software (FUJIFILM Toyama Chemical Co. Ltd., Tokyo, Japan; EXINI Diagnostics AB, Lund, Sweden). The present study aimed to determine the diagnostic ability of this software with an AI component to detect culprit coronary arteries on hybrid images.

\section{Methods}

\section{Patient population}

Table 1 shows the patient characteristics. The study group comprised 59 patients with stable CAD who had been assessed by coronary angiography within 60 days of MP-SPECT. All patients underwent stress MPI using adenosine at an infusion rate of $120 \mu \mathrm{g} / \mathrm{kg} / \mathrm{min}$ for $6 \mathrm{~min}$. At $3 \mathrm{~min}$ after starting the adenosine infusion, $250-370 \mathrm{MBq}$ of ${ }^{99 \mathrm{~m}} \mathrm{Tc}$-sestamibi (MIBI) (FUJIFILM Toyama Chemical Co. Ltd.) or tetrofosmin (Nihon Medi-Physics Co. Ltd., Tokyo, Japan) was injected. The rest of the study included $740-1110 \mathrm{MBq}$ of ${ }^{99 \mathrm{~m}} \mathrm{Tc}$-tetrofosmin or MIBI. The patients were assessed by SPECT approximately $60 \mathrm{~min}$ after injection. All patients underwent a single-day stress-rest protocol. 
Table 1 Demographics of patients assessed by MP-SPECT and invasive coronary artery angiography

\begin{tabular}{|c|c|}
\hline & Mean \pm SD or number $(n)$ \\
\hline Age (year) & $70.2 \pm 10.6$ \\
\hline Gender (M vs. F) & 43 vs. 14 \\
\hline Height (cm) & $162.5 \pm 10.4$ \\
\hline Weight (kg) & $61.9 \pm 13.6$ \\
\hline Body mass index $\left(\mathrm{kg} / \mathrm{m}^{2}\right)$ & $23.2 \pm 3.4$ \\
\hline Hypertension & $48(81.4 \%)$ \\
\hline Diabetes mellitus & $42(71.2 \%)$ \\
\hline Dyslipidemia & $34(57.6 \%)$ \\
\hline $\operatorname{eGFR}\left(\mathrm{mL} / \mathrm{min} / 1.73 \mathrm{~m}^{2}\right)$ & $61.6 \pm 27.0$ \\
\hline No significant stenosis & $20(33.9 \%)$ \\
\hline 1-vessel disease & $20(33.9 \%)$ \\
\hline 2-vessel disease & $11(18.6 \%)$ \\
\hline 3-vessel disease & $8(13.6 \%)$ \\
\hline \multicolumn{2}{|c|}{ Regions of vessels with $\geq 50 \%$ stenosis } \\
\hline LAD & $26(44.1 \%)$ \\
\hline LCX & $15(25.4 \%)$ \\
\hline RCA & $19(32.2 \%)$ \\
\hline \multicolumn{2}{|c|}{ Regions of vessels with $\geq 75 \%$ stenosis } \\
\hline LAD & $18(30.5 \%)$ \\
\hline LCX & $11(18.6 \%)$ \\
\hline RCA & $15(25.4 \%)$ \\
\hline SSS (LAD, LCX, RCA, total) & $2.3 \pm 2.8,3.1 \pm 3.4,2.7 \pm 3.1,7.8 \pm 6.8$ \\
\hline SRS (LAD, LCX, RCA, total) & $0.92 \pm 1.7,2.0 \pm 2.8,1.7 \pm 2.8,4.3 \pm 5.4$ \\
\hline SDS (LAD, LCX, RCA, total) & $1.2 \pm 1.7,1.0 \pm 1.3,1.1 \pm 1.5,3.4 \pm 3.1$ \\
\hline EDV $(\mathrm{mL})$ at rest & $98.4 \pm 60.3$ \\
\hline ESV $(\mathrm{mL})$ at rest & $50.3 \pm 54.0$ \\
\hline LVEF (\%) at rest & $57.6 \pm 17.2$ \\
\hline
\end{tabular}

Data are shown as means \pm SD or numbers $(n)$. EDV end-diastolic volume, eGFR estimated glomerular filtration rate, ESV end-systolic volume, $F$ female, $L A D$ left anterior descending coronary artery, $L C X$ left circumflex coronary artery, $L V E F$ left ventricular ejection fraction, $M$ male, $M P$ myocardial perfusion, $R C A$ right coronary artery, $S D$ standard deviation, $S D S$ summed difference score, SPECT single photon emission computed tomography, SRS summed rest score, SSS summed stress score

\section{MP-SPECT images}

\section{Acquisition}

Patients were assessed by MP-SPECT using a Symbia T6 SPECT-CT system (Siemens, Erlangen, Germany; Siemens Japan, Tokyo, Japan). The equipment consisted of a dual-head gamma camera in $180^{\circ}$ geometry equipped with a low-energy, highresolution (LEHR) collimator. The SPECT data were acquired in a $64 \times 64$ matrix using the following parameters: zoom, $\times 1.45$; pixel size, $6.6 \mathrm{~mm}$, duration, and $35 \mathrm{~s}$ per projection for a total acquisition period of $20 \mathrm{~min}$. We obtained a total of 60 frames, at $6^{\circ}$ per step over $360^{\circ}$. The cardiac cycle was divided into 16 frames for electrocardiography (ECG) gating. R-R intervals that averaged $\pm 25 \%$ on the ECG monitor were accepted for gating. The energy was centered at $140 \mathrm{keV}$, with a $15 \%$ window for SPECT/CT imaging. 
Scatter and attenuation were not corrected. The detectors were placed close to the patient in circular mode at a radius of $24-25 \mathrm{~cm}$.

\section{Reconstruction}

Data were reconstructed using a three-dimensional iterative method based on an ordered subset expectation maximization (3D-OSEM) algorithm with resolution recovery (RR; Flash 3D) and 120 updates (12 subsets $\times 10$ iterations). This algorithm is available in the e.soft application package (Siemens). A Gaussian filter was used in the OSEM algorithm, and RC was used for smoothing. The full width at half maximum of the Gaussian filter was $13.2 \mathrm{~mm}$.

\section{Analysis and display}

Gated SPECT images were automatically analyzed to calculate left ventricular (LV) volume and function, including end-diastolic volume (EDV), end-systolic volume (ESV), and left ventricular ejection fraction (LVEF), using quantitative gated SPECT (QGS) software (Cedars Sinai Medical Center, Los Angeles, CA, USA) and cardioREPO/EXINI Heart software. Myocardial perfusion defects were semi-quantified using a 17-segment model with 5-point visual scoring. The \%uptake by each segment was analyzed using quantitative perfusion SPECT (QPS) software (Cedars Sinai Medical Center). We used normal databases from the Japanese Society of Nuclear Medicine (JSNM) Working Group, which was created by the collaboration of multiple Japanese institutions (Nakajima et al. 2007). The databases have been used in Japanese clinical practice, and the population-specific JSNM database was superior to American database (Nakajima et al. 2009). Ischemia was defined as a summed difference score between stress and resting images of two or more.

\section{Hybrid images of MP-SPECT and CCTA}

Patients were assessed by CCTA using SOMATOM Definition Flash (Siemens Healthcare, Erlangen, Germany; Siemens Japan, Tokyo, Japan), a 128-section, dual X-ray source CT scanner. Images were acquired using CCTA after $\sim 60 \mathrm{~mL}$ of contrast medium (Iopamidol $370 \mathrm{mg} / \mathrm{mL}$, Iopamiron $370 \mathrm{mg} / \mathrm{mL}$; Bayer Yakuhin, Ltd., Osaka, Japan) was administered at a flow rate of $4 \mathrm{~mL} / \mathrm{s}$ followed by a $40 \mathrm{~mL}$ saline flush at the same rate. The scanning parameters were as follows: collimation, $128 \times 0.6 \mathrm{~mm}$; gantry rotation, $280 \mathrm{~ms}$; step-and-shoot mode; tube voltage, $120 \mathrm{kV}$; tube current of $340 \mathrm{mAs} /$ rotation; and a $220-\mathrm{mm}$ field of view. Axial images were reconstructed with a slice thickness of $0.75 \mathrm{~mm}$ and a reconstruction kernel of B35f (Heart View medium). All data were transferred to an Advantage Workstation 4.6 (GE Healthcare, Chicago, IL, USA; GE Healthcare Japan, Tokyo, Japan) and then manual registration of the CCTA and MP-SPECT images proceeded using pixel-shifting.

\section{Artificial neural network}

We used the computer-aided diagnostic software cardioREPO/EXINI Heart that was developed in Sweden and subsequently introduced into Japan (Nakajima et al. 2018). Version 1.1 of the artificial neural network (ANN) was trained on data derived from 1001 Japanese patients at 12 hospitals in Japan, and the diagnostic accuracy was 
validated in 364 patients (Nakajima et al. 2017). At least two Japanese nuclear cardiology specialist determined abnormal stress defects and stress-induced ischemia by consensus. Areas of possible perfusion abnormalities in stress and rest images (stress and rest defects, respectively) were segmented, and the ANN judged candidate regions based on 16 features extracted from the shape, extent, location, count, perfusion homogeneity, regional motion, wall thickening, and sex to give a probability of abnormality (ANN probability) (Nakajima et al. 2018). The cutoff value was defined as the ANN probability of $50 \%$.

\section{Image interpretation}

Figure 1 shows a flow chart of the image interpretation. A radiological technologist analyzed probabilities of perfusion abnormalities using the ANN and assigned the culprit coronary artery on hybrid images of CCTA and MP-SPECT. We defined the gold standard as the nuclear cardiology specialist interpretation with all available clinical information. Because patients with old myocardial infarction, percutaneous coronary intervention, and coronary artery bypass grafting were included, which is common in our clinical practice, the truth could not be simply defined by stenosis or fractional flow reserve (FFR). In addition, the AI was trained to learn specialist reading and not to identify possible stenosis or decreased flow reserve. The gold standard was, therefore, determined using coronary angiography and clinically available information including myocardial perfusion SPECT, CCTA, hybrid images, patient history, and subsequent clinical courses. Significant coronary artery disease was defined as stenosis in at least one coronary artery, as indicated by an FFR of 0.80 or less or stenosis diameter of $>70 \%$ using invasive coronary artery angiography or ischemia using hybrid images of CCTA and myocardial perfusion SPECT if FFR was not available. Ischemia using hybrid images of CCTA and myocardial perfusion SPECT was defined by inducible perfusion abnormality judged by two nuclear cardiology specialists. Two other nuclear medicine physicians evaluated the SPECT images, then the hybrid images of CCTA

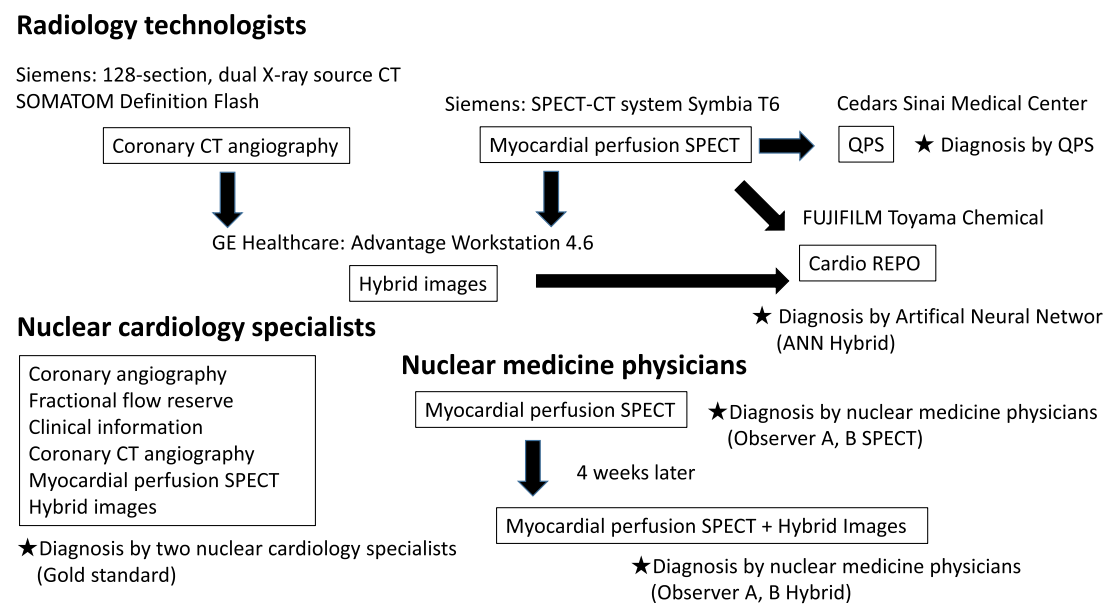

Fig. 1 Basic flow chart of roles of radiology technologists, nuclear medicine physicians, and interpretation. ANN artificial neural network, CT computed tomography, QPS quantitative perfusion single-photon emission computed tomography, SPECT single-photon emission computed tomography 
and MP-SPECT in the same way 4 weeks later, using four grades of confidence to evaluate their interpretation of regional abnormalities as follows: 1 , definitely normal; 2 , probably normal; 3 , probably abnormal; and 4 , definitely abnormal. This cutoff value was defined as 3 . The same physicians then drew the regions on polar maps and evaluated confidence in their interpretations of the culprit coronary artery as RCA, LCX, left anterior descending coronary artery (LAD), RCA or LCX (equivocal), LCX or LAD (equivocal), and LAD or RCA (equivocal). Diagnostic performance, including sensitivity, specificity, and accuracy in the detection of culprit coronary arteries, was compared among observer A, observer B, and the ANN.

\section{Statistical analysis}

Data were statistically analyzed using JMP version 12 (SAS Institute Inc., Cary, NC, USA). We evaluated CAD detectability based on areas under receiver operating characteristic (ROC) curves (AUC). All statistical tests were two-tailed, and values with $p<0.05$ were considered statistically significant.

\section{Results}

\section{Patient-based diagnosis}

Table 2 shows the likelihood ratios, Pearson statistics, and $p$ values for observers $\mathrm{A}, \mathrm{B}$, and the ANN. The abilities of the ANN and nuclear medicine physicians to diagnose infarction and/or ischemia were equivalent, and the ANN outperformed conventional semi-quantitative defect scoring using QPS (Fig. 2). The sensitivity, specificity, and accuracy of patient-based diagnoses by observers A and B using hybrid images were $85.0 \%, 72.2 \%$, and $81.0 \%$ and $73.1 \%, 73.2 \%$, and $78.0 \%$, respectively, and these values for the ANN and QPS were $87.8 \%, 72.2 \%$, and $84.7 \%$, and $73.2 \%, 77.8 \%$, and $74.6 \%$, respectively.

\section{Coronary artery-based diagnosis}

Figure 3 shows the sensitivity, specificity, and accuracy of coronary-based diagnosis by observer A, observer $\mathrm{B}$, the $\mathrm{ANN}$, and QPS in identifying culprits among the RCA, LAD, and LCX. Figures 4 and 5 show the AUC of observer A, observer B, the ANN, and QPS. The AUC were significantly improved using hybrid images in the RCA region (observer A, $p=0.0031$; observer $\mathrm{B}, p=0.042$ ). The culprit was most accurately diagnosed in the LAD region. The AUC to detect RCA lesions were better for CTA-guided myocardial perfusion than MP-SPECT image analysis. Figure 6 shows a patient with single-vessel disease of the RCA in which the ANN system interpreted the images of a detected ischemic area.

\section{Anatomical reference-based analysis}

Figure 7 showed the comparison of coronary artery-based diagnosis by observer A, observer B, ANN, and QPS in patients with MP-SPECT and hybrid images of CCTA and MP-SPECT when compared against anatomical reference standard defined as a stenosis diameter of $>70 \%$ by invasive coronary angiography. The AUC of observers A and B using hybrid images were 0.606 and 0.584 and using MP-SPECT were 0.576 and 0.546 , respectively. The AUC was not significantly improved using hybrid images. The 
Table 2 Contingency analysis of ANN and two observers

\begin{tabular}{|c|c|c|c|c|c|}
\hline & True findings & & & & \\
\hline & Infarction & Infarction + Ischemia & Ischemia & None & Total \\
\hline \multicolumn{6}{|l|}{ A. Observer A } \\
\hline Infarction & 10 & 4 & 1 & 1 & 16 \\
\hline Infarction + Ischemia & 2 & 4 & 6 & 0 & 12 \\
\hline Ischemia & 0 & 1 & 6 & 4 & 11 \\
\hline None & 3 & 2 & 2 & 13 & 20 \\
\hline Total & 15 & 11 & 15 & 18 & 59 \\
\hline \multicolumn{6}{|l|}{ B. Observer B } \\
\hline Infarction & 9 & 3 & 2 & 3 & 17 \\
\hline Infarction + Ischemia & 5 & 6 & 7 & 5 & 23 \\
\hline Ischemia & 1 & 1 & 5 & 3 & 10 \\
\hline None & 0 & 1 & 1 & 7 & 9 \\
\hline Total & 15 & 11 & 15 & 18 & 59 \\
\hline \multicolumn{6}{|l|}{ C. ANN } \\
\hline Infarction & 10 & 0 & 1 & 2 & 13 \\
\hline Infarction + Ischemia & 3 & 9 & 5 & 0 & 17 \\
\hline Ischemia & 0 & 0 & 7 & 1 & 8 \\
\hline None & 2 & 2 & 2 & 15 & 21 \\
\hline Total & 15 & 11 & 15 & 18 & 59 \\
\hline \multicolumn{6}{|l|}{ D. Statistical significance } \\
\hline & Likelihood ratio & $p$ value & Pearson statistics & $p$ value & \\
\hline ANN & 65.6 & $<0.0001$ & 68.1 & $<0.0001$ & \\
\hline Observer A & 44.8 & $<0.0001$ & 41.1 & $<0.0001$ & \\
\hline Observer B & 22.1 & 0.0087 & 22.4 & 0.0076 & \\
\hline
\end{tabular}

The likelihood ratios, Pearson statistics, and $p$ values for observer A (A), observer B (B), and ANN (C) Likelihood ratios, Pearson statistics, and probability $(p)$ estimates for observers $A, B$, and artificial neural network (ANN)



Fig. 2 Areas under ROC curves derived from patient-based analysis by ANN, two observers (a), and QPS (b). ANN artificial neural network, QPS quantitative perfusion SPECT, ROC receiver operating characteristics 


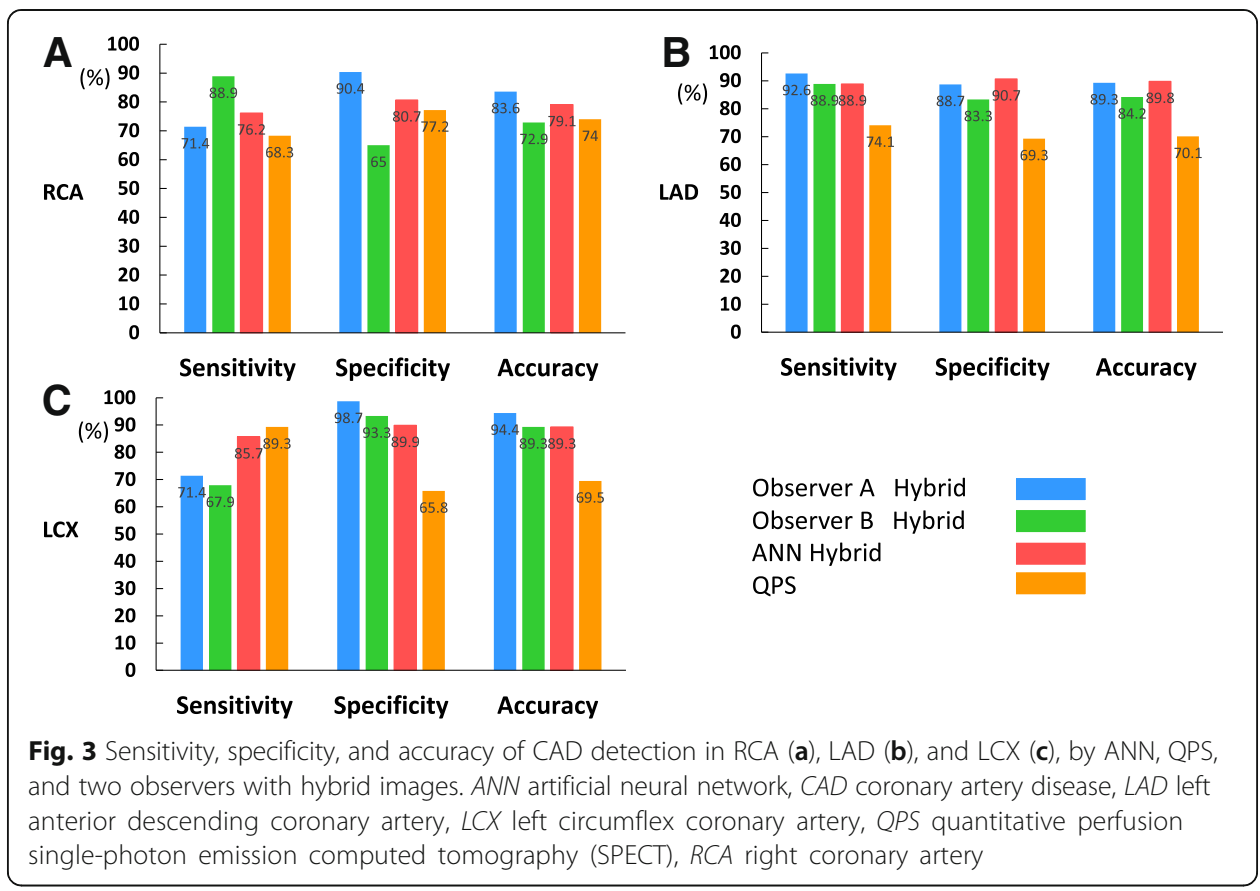

AUC of ANN and QPS were 0.568 and 0.544 , respectively. The AUC was not significantly improved using ANN.

\section{Discussion}

This study demonstrated that hybrid images of CCTA and MP-SPECT with neural network guidance could identify perfusion abnormalities effectively. When a radiology technologist analyzed hybrid images and MP-SPECT images using AI-based software to identify a culprit coronary artery, the diagnostic ability of the ANN was comparable to that of nuclear medicine physicians and outperformed conventional semi-quantitative defect scoring.

\section{The need for applying Al software}

MPI has been interpreted based on integrated understanding of myocardial perfusion distribution at stress and rest, and comparison between these two conditions using visual analysis and automatic scoring contributed to diagnosis of ischemia in clinical practice. However, while typical perfusion defects or ischemia could be unanimously judged as abnormal, nuclear physicians sometimes struggle to diagnose for equivocal findings; in such situation, a method to support objective and quantitative interpretation is considered useful. Nuclear medicine physicians tend to intentionally overlook or over-read MPI findings based on the experience of previous success or failure of interpretation. On the other hand, the ANN could emphasize possible abnormal regions with sufficient degrees of certainty and reproducibility. Since cardiologists and radiologists are not always specialists in nuclear cardiology, such ANN-based suggestions might enhance diagnostic confidence. The probability of abnormalities 


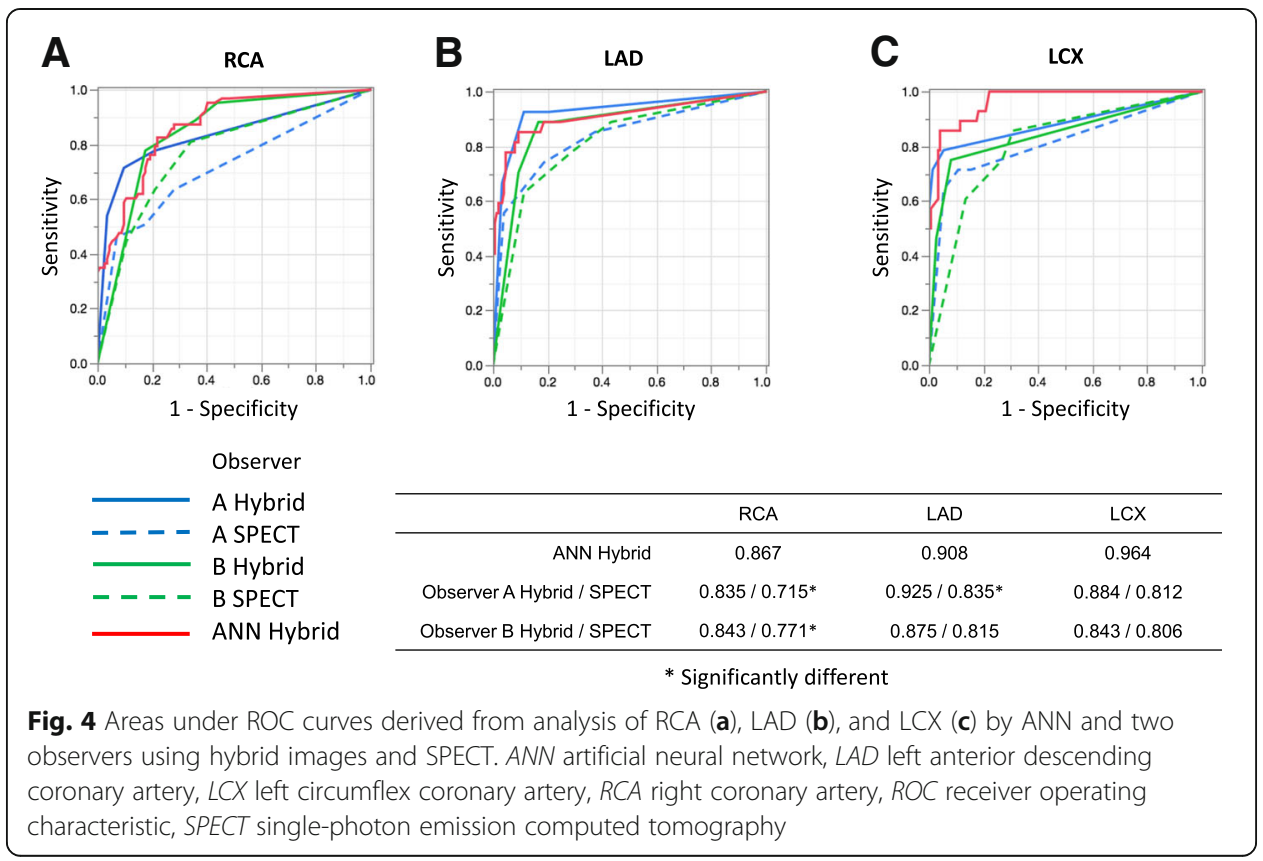

provided by the ANN might be useful reference even for nuclear cardiology specialist. When a radiology technologist analyzed hybrid images and MP-SPECT images using AI-based software to identify a culprit coronary artery, the diagnostic ability of the ANN was comparable to that of nuclear medicine physicians and outperformed conventional semi-quantitative defect scoring. This finding was consistent with those of other studies (Nakajima et al. 2018; Nakajima et al. 2017; Nakajima et al. 2015; Johansson et al. 2014).

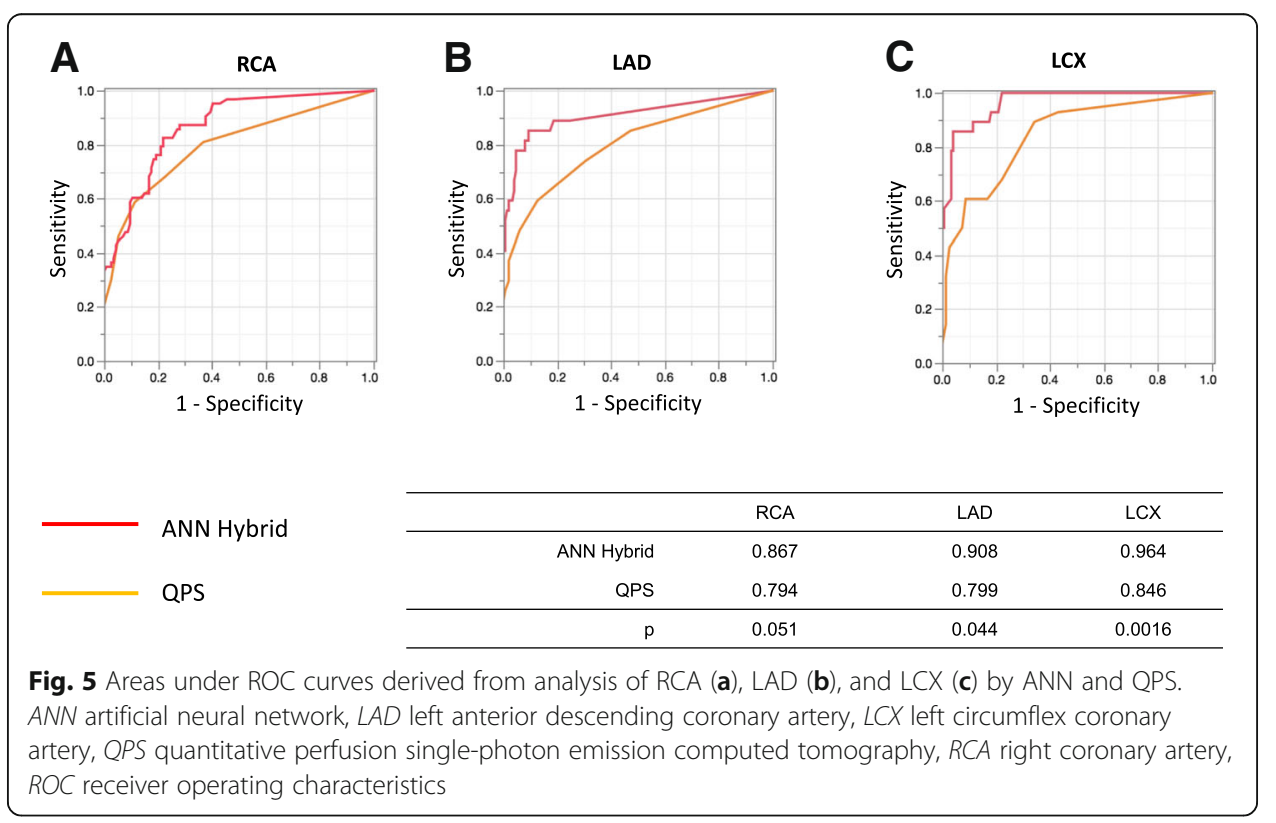




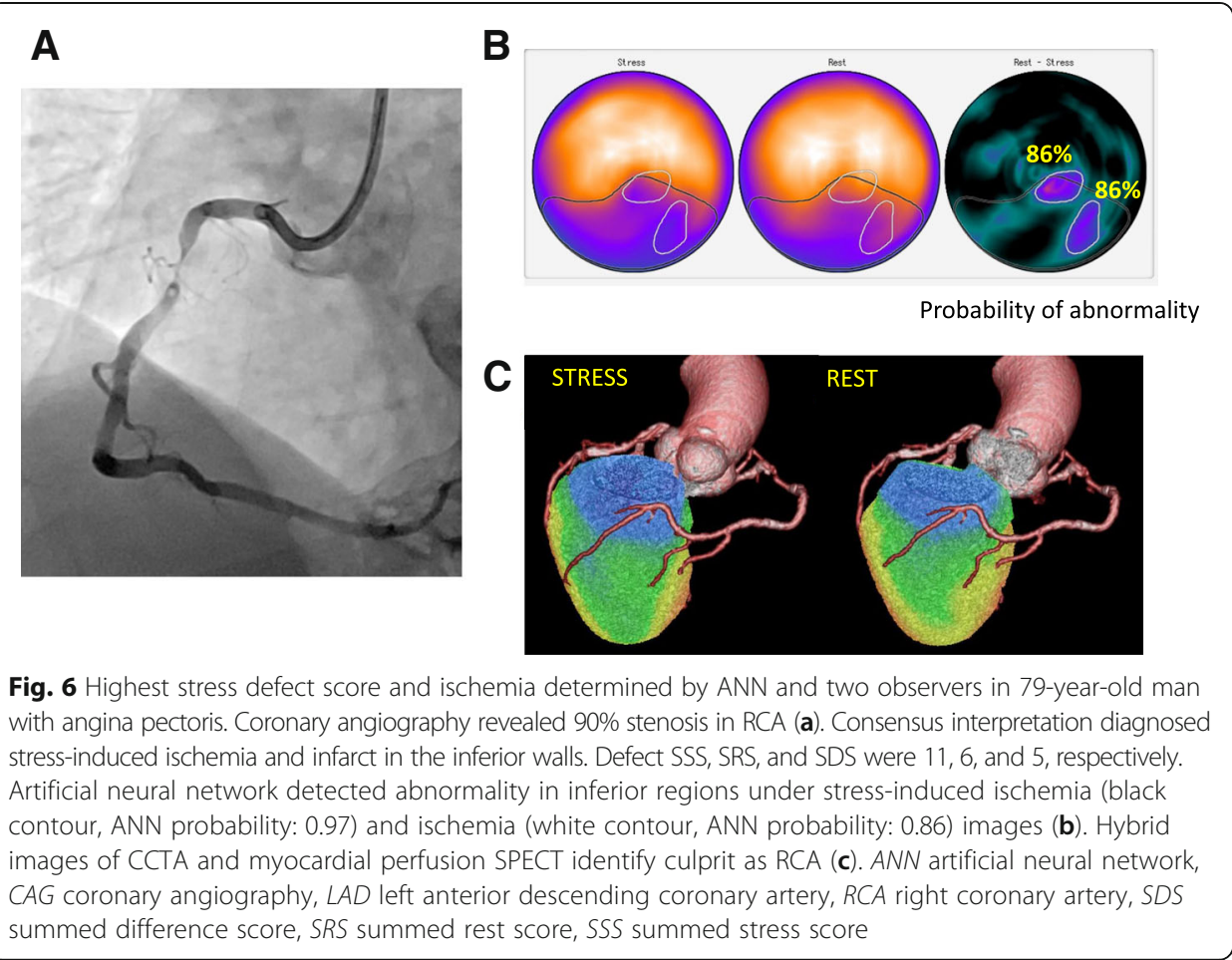

\section{Identification of coronary artery lesions}

Our analysis of diagnostic performance in specific vessels showed that CCTA-guided quantitation was useful in the RCA territory, and that the diagnostic accuracy was comparable in the LAD territory between nuclear physicians and software-guided technologist interpretation. Clinicians must accurately match the anatomy of the coronary artery with the location of an abnormality in SPECT images to accurately assess myocardial ischemia. Generally, the LCX flows orthogonally to the LAD from the 11 o'clock position in polar maps. The obtuse marginal and posterolateral branches at 2-3 and 4-5 o'clock respectively emerge from the LCX in the left atrioventricular groove. The RCA runs in the right atrioventricular groove and sends out the posterior descending artery at 7 o'clock. The atrioventricular node artery branches from the posterior descending artery toward the 5-6 o'clock region (Nagano et al. 2016). However, using only SPECT to assign coronary artery territories is difficult because perfused regions of the LCX and RCA vary among individuals. Therefore, CCTA is needed to accurately identify culprit coronary arteries in patients with ischemia.

\section{Accuracy of MP-SPECT to detect coronary artery disease}

Nakajima et al. reported that the sensitivity, specificity, and accuracy of interpreting culprit coronary artery territories using MP-SPECT without hybrid images were of 77\%, 74\%, and 75\%, respectively (Nakajima et al. 2010). Quantitative analysis based on summed QPS stress scores of $\geq 4$ was used as threshold of abnormality; Johansson et al. reported that the specificity of computer-aided diagnostic software was higher than that of summed stress scores $(86.8 \%$ vs. $73.6 \% ; p<0.001)$ at the same level of sensitivity. Danad et al. reported that quantitative analysis based on summed QPS 


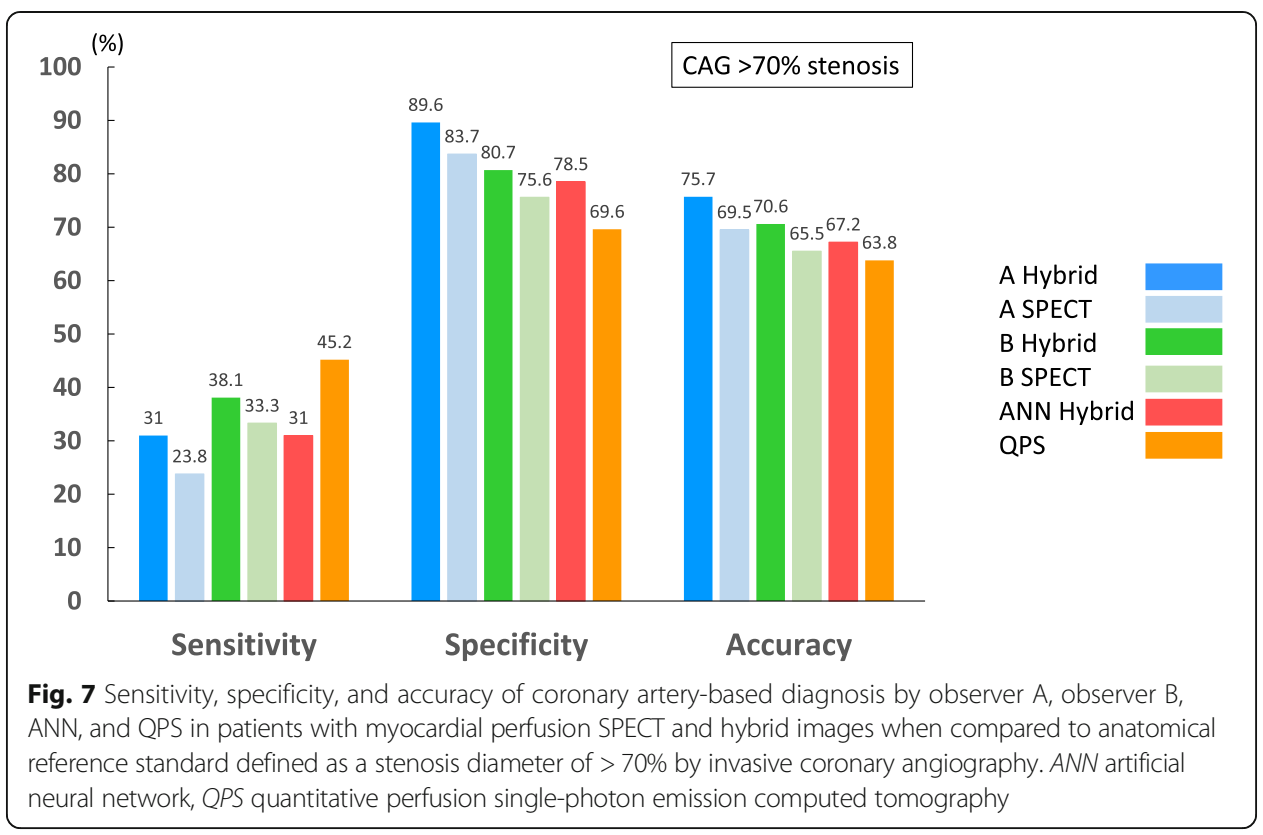

difference scores $\geq 2$ conferred an overall sensitivity, specificity, and accuracy of hybrid SPECT and CCTA for diagnosis of ischemia-causing coronary artery disease as indicated by FFR were 50\%, 97\%, and 76\% (Danad et al. 2017). Although the diagnostic ability was dependent on the patient selection criteria, these results of diagnostic ability were comparable to our finding and support our conclusions.

\section{Reduction of artifacts}

Since the ANN does not consider body motion, body attenuation, or artifacts, these factors need to be reduced. Body motion tends to result in misdiagnosis, particularly when using the ANN. Although this can be addressed using motion correction software, reacquisition should proceed when large amounts of motion are detected by linography and/or sinography. Activity in sub-diaphragmatic organs can cause artifacts in images by simulating perfusion defects and concealing true defects. Iterative reconstruction suppresses streak artifacts caused by high extracardiac activity such as gallbladder activity. Reacquisition should also be considered when the extracardiac uptake of ${ }^{99 \mathrm{~m}} \mathrm{Tc}$ is more intense than cardiac uptake, or when extracardiac uptake is not distinguishable from the inferior cardiac wall. Importantly, the ANN might not detect widespread, very mild ischemia in poor-quality images. Less noisy images are generally expected, even with limited radiation doses. Thus, dose and acquisition time should be balanced.

\section{Disadvantages of hybrid images of CCTA and MP-SPECT}

Although hybrid images of CCTA and MP-SPECT improves the detection of coronary artery disease and enhances diagnostic confidence, this procedure has not yet become routine in the clinical setting at many hospitals. The hybrid images of CCTA and MP-SPECT can require time-consuming manual intervention (Piccinelli et al. 2018). Radiation doses delivered to patients should also be considered. Coronary arteries can 
be directly visualized by CCTA and coronary atherosclerosis can be detected and quantified. Individual coronary plaques can be identified, and information can be gathered regarding the total extent, severity, location, and composition of coronary artery disease (van Rosendael et al. 2017). However, a phantom study found that the radiation dose of 128-section, dual-source CT coronary angiography was $195.7 \mathrm{mGy}$ in the low-pitch spiral mode and $96.1 \mathrm{mGy}$ in the step-and-shoot mode (Matsubara et al. 2012). Thus, the latter mode should initially be applied because less radiation is absorbed.

\title{
Study limitations
}

This single-center, retrospective, observational study has several limitations. We did not measure FFR in all patients and the cohort of patients was quite small. It was difficult to measure FFR in all cases and all stenotic coronary arteries. Therefore, data selection was biased by the clinical conditions that prompted the need for invasive angiography, SPECT, and CCTA, as only a minority of patients are evaluated for CAD using these modalities.

We did not apply fully automated quantitative analysis or image registration; the contour definitions and vascular territories were manually guided by co-registered CCTA anatomy.

\section{Conclusions}

Hybrid images of CCTA and MP-SPECT improved the ability to identify culprit coronary arteries in patients with stable ischemic heart disease. The diagnostic abilities of the ANN and nuclear medicine physicians were comparable. Because of interindividual differences in the perfused regions of the RCA and LCX, to identify a coronary artery responsible for perfusion defects of the inferior wall is difficult without using hybrid images.

\begin{abstract}
Abbreviations
3D-OSEM: Three-dimensional iterative method based on ordered subset expectation maximization; Al: Artificial intelligence; ANN: Artificial neural network; AUC: Area under ROC curve; CAD: Coronary artery disease; CCTA: Coronary computed tomography angiography; ECG: Electrocardiography; EDV: End-diastolic volume; ESV: End-systolic volume; FFR: Fractional flow reserve; LAD: Left anterior descending coronary artery; LCX: Left circumflex coronary artery; LV: Left ventricular; LVEF: Left ventricular ejection fraction; MPI: Myocardial perfusion imaging; QGS: Quantitative gated SPECT; QPS: Quantitative perfusion SPECT; RCA: Right coronary artery; ROC: Receiver operating characteristics; RR: Resolution recovery; SPECT: Single-photon emission computed tomography
\end{abstract}

\footnotetext{
Acknowledgements

Authors thank Norma Foster for editorial assistance. We are grateful to K. Sakuda, H. Nunome, A. Mizutani, Y. Koshino, and all radiology technologists at Kanazawa University Hospital for creating hybrid images and providing technical support.

\section{Funding}

Kanazawa University Hospital provided funding for the design of the study, the collection, analysis, and interpretation of data and manuscript preparation.
}

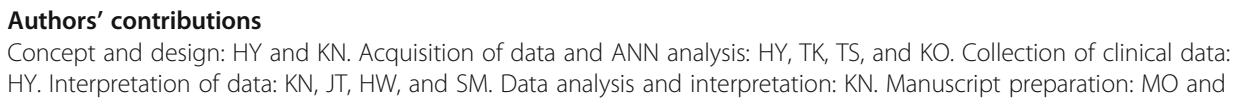


Competing interests

K. Nakajima has collaborated with FUJIFILM Toyama Chemical, Tokyo, Japan to develop software.

\section{Publisher's Note}

Springer Nature remains neutral with regard to jurisdictional claims in published maps and institutional affiliations.

\section{Author details}

'Department of Radiological Technology, Kanazawa University Hospital, 13-1 Takara-machi, Kanazawa 920-8641, Japan. ${ }^{2}$ Department of Nuclear Medicine, Kanazawa University Hospital, Kanazawa, Japan. ${ }^{3}$ Department of Physics, Kanazawa Medical University, Kahoku, Japan. ${ }^{4}$ Department of Quantum Medical Technology, Institute of Medical, Pharmaceutical and Health Sciences, Kanazawa University, Kanazawa, Japan.

Received: 19 November 2018 Accepted: 19 February 2019

Published online: 18 March 2019

\section{References}

Arsanjani R, Dey D, Khachatryan T, Shalev A, Hayes SW, Fish M et al (2015) Prediction of revascularization after myocardial perfusion SPECT by machine learning in a large population. J Nucl Cardiol 22:877-884

Arsanjani R, Xu Y, Dey D, Fish M, Dorbala S, Hayes S et al (2013a) Improved accuracy of myocardial perfusion SPECT for the detection of coronary artery disease using a support vector machine algorithm. J Nucl Med 54:549-555

Arsanjani R, Xu Y, Dey D, Vahistha V, Shalev A, Nakanishi R et al (2013b) Improved accuracy of myocardial perfusion SPECT for detection of coronary artery disease by machine learning in a large population. J Nucl Cardiol 20:553-562

Danad I, Raijmakers PG, Driessen RS, Leipsic J, Raju R, Naoum C et al (2017) Comparison of coronary CT angiography, SPECT, PET, and hybrid imaging for diagnosis of ischemic heart disease determined by fractional flow reserve. JAMA Cardiol 10: $1100-1107$

Guner LA, Karabacak NI, Akdemir OU, Karagoz PS, Kocaman SA, Cengel A et al (2010) An open-source framework of neural networks for diagnosis of coronary artery disease from myocardial perfusion SPECT. J Nucl Cardiol 17:405-413

Hachmovitch R, Hayes SW, Friedman JD, Cohen I, Berman DS et al (2003) Comparison of the short-term survival benefit associated with revascularization compared with medical therapy in patients with no prior coronary artery disease undergoing stress myocardial perfusion single photon emission computed tomography. Circulation 107:2900-2906

Henzlova MJ, Duvall WL, Einstein AJ, Travin MI, Verberne HJ (2016) ASNM imaging guidelines for SPECT nuclear cardiology procedures: stress, protocols, and tracers. J Nucl Cardiol 23:606-609

Johansson L, Edenbrandt L, Nakajima K, Lomsky M, Svensson SE, Tragardh E (2014) Computer-aided diagnosis system outperforms scoring analysis in myocardial perfusion imaging. J Nucl Cardiol 21:416-423

Kirisli HA, Gupta V, Shahzad R, Al Younis I, Dharampal A, Geuns RJ et al (2014) Additional diagnosis value of integrated analysis of cardiac CTA and SPECT MPI using the SMARTVis system in patients with suspected coronary artery disease. J Nucl Med 55:55-57

Kiriyama T, Fukushima Y, Hayashi H, Takano H, Kumita S (2018) Feasibility of combined risk stratification with coronary CT angiography and stress myocardial SPECT in patients with chronic coronary artery disease. Ann Nucl Med 32:22-33

Kiso K, Tateishi E, Imoto A, Terakawa Y, Murakawa K, Fukuda T (2017) The feasibility of quantitative assessment of myocardial perfusion using single photon emission computed tomography imaging: current status and future challenges. Ann Nucl Cardiol 3:167-171

Levine GN, Bates ER, Blankenship JC, Bailey SR, Bittl JA, Cercek B et al (2011) 2011 ACCF/AHA/SCAl guideline for percutaneous coronary intervention. A report of the American College of Cardiology Foundation/American Heart Association Task Force on practice guidelines and the society for cardiovascular angiography and interventions. Am Coll Cardiol 58:e44-e122

Matsubara K, Koshida H, Sakata K, Takata T, Horii J, lida H et al (2012) Radiation dose and physical image quality in 128section dual-source computed tomographic coronary angiography: a phantom study. J Appl Clin Med Phys 13:252-261

Matsuda A, Takeishi Y (2016) Choosing the appropriate examination for diagnosis of stable ischemic heart disease. Ann Nucl Cardiol 2:167-173

Matsumoto N, Hirayama A (2017) Nuclear medicine is the best approach for detecting coronary artery disease: from JSNC 2016 evening seminar. Ann Nucl Cardiol 3:150-154

Motowani M, Dey D, Berman DS, Germano G, Achenbach S, Al-Mallah MH et al (2016) Machine learning for prediction of allcause mortality in patients with suspected coronary artery disease: a 5-year multicentre prospective registry analysis. Eur Heart J 38:500-507

Nagano N, Hashimoto A, Miura T (2016) Basics of reading myocardial perfusion SPECT to identify coronary artery lesions. Ann Nucl Cardiol 2:158-161

Nakajima K, Kudo T, Nakata T, Kiso K, Kasai T, Taniguchi Y et al (2017) Diagnostic accuracy of an artificial neural network compared with statistical quantitation of myocardial perfusion images: a Japanese multicenter study. Eur J Nucl Med 44:2280-2283

Nakajima K, Kumita S, Ishida Y, Momose M, Hashimoto J, Morita K et al (2007) Creation and characterization of Japanese standards for myocardial perfusion SPECT: database from Japanese Society of Nuclear Medicine Working Group. Ann Nucl Med 21:505-511

Nakajima K, Matsuo S, Kawano M, Matsumoto N, Hashimoto J, Yoshinaga K et al (2010) The validity of multi-center common normal database for identifying myocardial ischemia: Japanese society of nuclear medicine working group database. Ann Nucl Med 24:99-105

Nakajima K, Matsuo S, Wakabayashi H, Yokoyama K, Bunko H, Okuda K et al (2015) Diagnostic performance of artificial neural network for detecting ischemia in myocardial perfusion imaging. Circ J 79:1549-1556 
Nakajima K, Okuda K, Kawano M, Matsuo S, Slomka P, Germano G et al (2009) The importance of population-specific normal database for quantification of myocardial ischemia: comparison between Japanese 360 and 180-degree databases and a US database. J Nucl Cardiol 16(3):422-430

Nakajima K, Okuda K, Watanabe S, Matsuo S, Kinuya S, Toth K et al (2018) Artificial neural network retrained to detect myocardial ischemia using a Japanese multicenter database. Ann Nucl Med 32:303-310

Nanasato M, Matsumoto N, Nakajima K, Chikamori T, Moroi M, Takehana K et al (2018) Prognostic impact of reducing myocardial ischemia identified using ECG-gated myocardial perfusion SPECT in Japanese patients with coronary artery disease: J-ACCESS 4 study. Int J Cardiol 267:202-207

Piccinelli M, Cooke DC, Garcia EV (2018) Multimodality image fusion for coronary artery disease detection: concepts and latest developments. Ann Nucl Cardiol 4:74-78

Sato A, Makiguchi N, Narita K, Brugada J, Boriani G, Bremerich J et al (2015) Cardiovascular imaging for nuclear cardiologists: first step of coronary computed tomography. Ann Nucl Cardiol 1:69-76

Shaw LJ, Berman DS, Marron DJ, Mancini GB, Hayes SW, Hartigan PM et al (2008) Optimal medical therapy with or without percutaneous coronary intervention to reduce ischemic burden: results from the clinical outcomes utilizing revascularization and aggressive drug evaluation (COURAGE) trial nuclear sub study. Circulation 117:1283-1291

Slomka PJ, Cheng VY, Dey D, Woo J, Ramesh A, Van Kriekinge S et al (2009) Quantitative analysis of myocardial perfusion SPECT anatomically guided by coregistered 64-slice coronary CT angiography. J Nucl Med 50:1621-1630

van Rosendael AR, Smit J, Scholte A (2017) Coronary CTA is the best approach to detect coronary artery disease. Ann Nucl Cardiol 3:137-142

Submit your manuscript to a SpringerOpen ${ }^{\circ}$ journal and benefit from:

- Convenient online submission

- Rigorous peer review

- Open access: articles freely available online

- High visibility within the field

Retaining the copyright to your article

Submit your next manuscript at $>$ springeropen.com 